\title{
Les propriétés antidépressives de la kétamine
}

\section{Une revue}

> La dépression majeure est une affection fréquente. Malgré des avancées importantes en psychopharmacologie depuis les années 1950, le délai d'action et la résistance aux traitements demeurent des défis thérapeutiques pour les agents traditionnels à action antidépressive, tels que les bloqueurs de recapture de la sérotonine. La découverte récente de l'effet antidépresseur rapide de la kétamine, un antagoniste des récepteurs NMDA (N-méthyl-D-aspartate), a révolutionné la recherche dans ce domaine. Cet article offre une revue compréhensive de la recherche clinique portant sur les effets antidépresseurs de la kétamine ainsi que sur ses mécanismes d'action présumés. <

\section{La dépression et les défis de son traitement}

La dépression majeure est une affection fréquente, avec une prévalence à vie estimée à $11,3 \%$ [1]. Au niveau mondial, elle se retrouve au $1^{\text {er }}$ rang des maladies responsables d'années perdues dues à une invalidité [2]. La compréhension des mécanismes à l'origine de la dépression a beaucoup évolué depuis l'élaboration du modèle monoaminergique de la dépression, établi à la suite de la découverte des premiers agents à action antidépressive dans les années 1950. Selon ce modèle, un déficit de certains neurotransmetteurs, les monoamines (sérotonine, noradrénaline et dopamine), serait à l'origine des symptômes dépressifs. Sa compréhension a ainsi permis de développer des médicaments ciblant des éléments neuronaux précis, tels que la fluoxétine (Prozac $\left.{ }^{\circledR}\right)$, inhibiteur sélectif de recapture de la sérotonine (ISRS) [3]. Depuis, de nombreux médicaments agissant sur les systèmes monoaminergiques ont vu le jour. L'étude des mécanismes d'action des psychotropes s'est poursuivie, permettant de mieux comprendre l'interaction entre les différentes voies monoaminergiques [4] et de caractériser le profil pharmacodynamique de chaque molécule. Leur action (agoniste, antagoniste) et leur force de liaison vis-à-vis des nombreuses cibles moléculaires ont ainsi été décrites.

Vignette (Photo (c) Inserm - Constans, Jean-Marc).

\section{Charles Y. Desfossés ${ }^{1}$, Pierre Blier ${ }^{1-3}$}

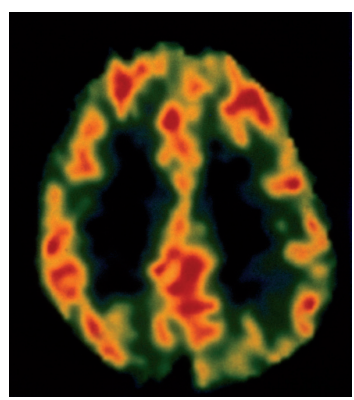

${ }^{1}$ The Royal's Institute of Mental Health Research, 1145 Carling Avenue, Ottawa, ON, K1Z 7K4, Canada.

${ }^{2}$ Department of Psychiatry, University of Ottawa, 1145 Carling Avenue, Ottawa, ON, KlZ $7 \mathrm{~K} 4$, Canada.

${ }^{3}$ Department of Cellular and Molecular Medicine, University of Ottawa, 451 Smyth Road, Ottawa, 0N, KIH 8M5, Canada. pierre.blier@theroyal.ca charles.desfosses.1@ulaval.ca

Malgré ces avancées importantes, la résistance au traitement et le délai d'action thérapeutique sont demeurés des enjeux majeurs. Dans la plus grande étude de pharmacothérapie pour le traitement de la dépression publiée à ce jour, environ le tiers des patients n'atteignait pas la rémission après 2 mois [5]. Généralement, il est en effet nécessaire d'attendre plusieurs semaines pour voir apparaître un effet antidépresseur marqué du traitement, ce qui conduit le patient à souffrir plus longtemps, en plus d'augmenter le risque de complications biologiques et sociales de la dépression.

Face à ces défis, la recherche de nouveaux modèles explicatifs et de nouvelles voies thérapeutiques s'est poursuivie. Au cours des années 1990, un effet antidépresseur des antagonistes des récepteurs NMDA (N-méthyl-D-aspartate) a été alors montré dans des modèles animaux de dépression, et il a été démontré que l'administration de médicaments efficaces dans la dépression tels que les agents tricycliques et les ISRS pouvaient moduler les récepteurs NMDA. Ces observations ont conduit à l'hypothèse que la modulation des récepteurs NMDA pourrait représenter la voie finale commune des différents agents à propriétés antidépressives [6], pavant ainsi la voie vers la découverte de l'action thérapeutique de la kétamine (voir Encadré).

\section{La kétamine, un agent à action antidépressive}

La démonstration initiale des effets antidépresseurs de la kétamine s'est faite progressivement, de façon plutôt inhabituelle dans une population de patients résistants au traitement. D'abord administrée en unidoses dans les études, la kétamine a montré un effet antidépresseur rapide et robuste mais non soutenu dans le temps. Cependant, des études de doses répétées, étalées sur une période de quelques semaines, ont ensuite révélé qu'il était possible de prolonger 


\section{Qu'est-ce que la kétamine?}

La kétamine, un antagoniste des récepteurs glutamatergiques NMDA ( $N$-méthyl-D-aspartate), a été développée dans les années 1960 par la compagnie Parke Davis à partir d'un dérivé de la PCP (phéncyclidine) dans un effort de développement d'un agent anesthésique intraveineux [34]. Elle a été approuvée par la FDA (Food and Drug Administration) en 1970 comme agent anesthésique dissociatif. Dans l'espèce humaine, elle est utilisée comme anesthésique dans des situations d'urgence pour des traumatismes, des brûlures et pour des procédures pédiatriques [34]. La kétamine a une demi-vie d'environ 2-4 heures en incluant ses métabolites. II existe deux énantiomères actifs de la kétamine, la S-kétamine et la R-kétamine, qui ont des propriétés pharmacocinétiques semblables, mais des propriétés pharmacodynamiques différentes. La principale voie d'administration est la voie intraveineuse (IV), quoiqu'il existe d'autres formulations pour d'autres voies : orale, rectale, intranasale et intramusculaire.

et même d'améliorer la réponse clinique. Il est important de mentionner que l'utilisation de la kétamine pour traiter la dépression demeure encore, à ce jour, hors monographie.

\section{Les études unidoses}

En 2000, la première étude clinique randomisée-contrôlée ( $E C R$ ) en double aveugle (DA) a utilisé un schéma en chassé-croisé ${ }^{1}$, dans lequel chaque participant recevait à une semaine d'intervalle et en alternance deux infusions sur 40 minutes, soit une infusion de kétamine $(0,5 \mathrm{mg} / \mathrm{kg})$ et une infusion de placébo [7]. Un effet antidépresseur statistiquement significatif de la kétamine par rapport au placébo a été observé dés 240 minutes après le traitement, et a atteint un maximum après 3 jours; sur les 8 patients traités par la kétamine, 7 ont eu une amélioration de leurs symptômes d'au moins $30 \%$ et 4 d'au moins $50 \%$ (pour les patients ayant reçu le placébo, seul un patient a vu ses symptômes s'améliorer). Toutefois, sur 7 patients en amélioration ayant terminé l'étude, 6 ont eu une rechute après 1 à 2 semaines.

En 2006, Zarate et al. [8] ont réalisé la première étude de réplication des résultats obtenus par le groupe de l'université de Yale (New Haven, États-Unis) [7]. Dans cette étude, parmi les 17 patients ayant reçu la kétamine, $71 \%$ et $29 \%$ ont atteint respectivement une réponse et une rémission (versus $0 \%$ avec le placébo) : l'effet significatif de la kétamine s'est révélé après 110 minutes de traitement et jusqu'à la fin du suivi de 7 jours post-infusion. Toutefois, une semaine après, seuls $35 \%$ des patients atteignaient encore le seuil de réponse clinique.

En 2010, Diazgranados et al. ont publié la première étude portant sur un traitement par la kétamine de la dépression bipolaire [9]. Alors que les deux premières études requéraient que les patients ne prennent aucun autre psychotrope, les patients de cette étude devaient présenter une absence de réponse à une dose thérapeutique de lithium ou d'acide valproïque, deux agents utilisés dans le traitement du trouble

${ }^{1}$ Le schéma en chassé-croisé est un schéma où chaque participant reçoit l'agent actif ET le placébo, en alternance. La randomisation se fait pour déterminer lequel des deux traitements sera reçu en premier. Ce type de schéma permet notamment de comparer chaque participant à lui-même, en plus de comparer les participants entre eux. bipolaire. Là encore ici, $71 \%$ des patients ayant reçu la kétamine ont atteint une réponse clinique ( $6 \%$ avec le placébo). L'effet antidépresseur était maximal deux jours après le traitement, le temps moyen de rechute étant de 6,8 jours. Cette étude a été répliquée en 2012 avec des résultats similaires [10].

Dans une sous-analyse de patients inclus dans une autre étude, Ibrahim et al. [11] ont montré qu'une dose unique de kétamine améliorait les symptômes dépressifs, avec un effet modéré chez les patients pour lesquels l'électroconvulsivothérapie (ECT) avait échoué $(n=17)$, mais avec un effet important pour ceux n'y ayant pas été exposés $(n=23)$. Ces résultats sont concordants avec ceux montrant, dans un échantillon de 150 patients résistants aux $\varepsilon C T$, une réponse à des traitements répétés de kétamine, et un maintien de cette réponse après un an de traitement chez $37 \%$ d'entre eux [12].

Murrough et al. [13] ont été les premiers à utiliser en 2013, un placébo «actif», la benzodiazépine midazolam. Dans cette étude, 73 patients présentant une dépression résistante au traitement ont reçu (après randomisation $2: 1)^{2}$ une infusion unique soit de kétamine $(0,5 \mathrm{mg} / \mathrm{kg})$ soit de midazolam $(0,045 \mathrm{mg} / \mathrm{kg})$. Après $24 \mathrm{~h}$, une réponse clinique a été obtenue chez $64 \%$ des patients traités par la kétamine (versus $28 \%$ avec le placébo «actif »).

Mentionnons finalement que plusieurs méta-analyses ont confirmé l'efficacité transitoire d'une dose unique de kétamine. L'une d'elles, publiée en 2015 [14], inclut les patients de 7 études randomisées contrôlées, pour un groupe total de 147 patients ayant reçu la kétamine. Elle démontre un rapport de cotes (odds ratio en anglais $\left.{ }^{3}\right)$ à 24 heures de $9,87(4,37-22,29)$ et 14,47 $(2,67-78,49)$, respectivement pour la réponse et la rémission. Le rapport de cotes diminue progressivement mais demeure statistiquement significatif jusqu'à 2 semaines post-infusion.

\section{Les études multidoses à court-terme}

$\varepsilon$ n raison de la rechute précoce des symptômes dépressifs observée dans les études précédentes, la prolongation et le maintien de la réponse antidépressive après traitement par la kétamine sont devenus l'objet de nombreux travaux à partir de 2010 .

Les deux premières études de maintien $[15,16]$ consistaient en l'administration de 6 infusions de kétamine $(0,5 \mathrm{mg} / \mathrm{kg})$, en ouvert ${ }^{4}, 3$ fois par semaine durant 2 semaines. Pour 24 patients sans autre médication

\footnotetext{
Deux patients traités par la kétamine pour un patient traité par le midazolam. 3 C'est-à-dire la force du lien entre l'exposition à la kétamine et l'amélioration. ${ }^{4}$ C'est-à-dire sans que les patients, les évaluateurs ou les médecins soient en
} aveugle. En anglais, open-label. 

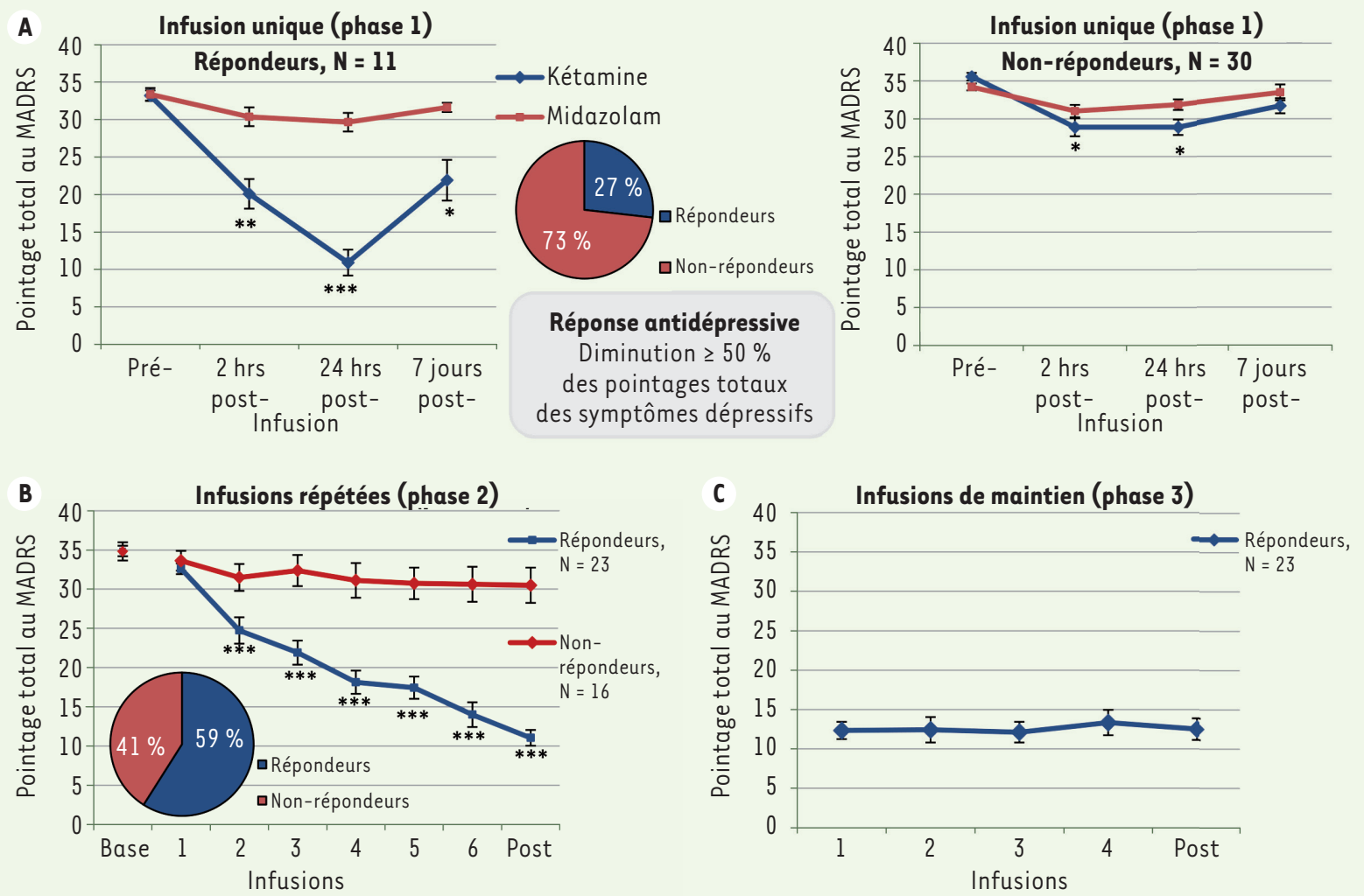

Figure 1. A. Effets d'une dose unique de kétamine $(0,5 \mathrm{mg} / \mathrm{kg})$ versus placébo, chez les répondeurs et non-répondeurs. $\boldsymbol{B}$. Effets cumulatifs des infusions répétées de kétamine lors de la phase 2 , chez les répondeurs et non-répondeurs. C. Phase 3 : effet des infusions de maintien hebdomadaires chez les répondeurs de la phase 2. MADRS: Montgomery-Åsberg Depression Rating Scale. ${ }^{*}=p<0.05 . * \star=p<0.01 . * \star *=p<0.001$ (adapté de [23]).

psychotrope, un taux de réponse de $71 \%$ a été observé à la fin des 2 semaines ; $94 \%$ des répondeurs avaient déjà atteint une réponse clinique 4 heures après la première infusion. Le temps moyen de rechute dépressive était de 18 et 19 jours.

Six autres études ont utilisé des protocoles légèrement différents pour explorer la réponse à des infusions répétées, variant entre 3 et 6 infusions de kétamine $(0,5 \mathrm{mg} / \mathrm{kg})$ de 40,45 ou 100 minutes, étalées sur une période de 2 à 3 semaines [17-22]. Une de ces études a utilisé une stratégie d'augmentation de dose, passant de 0,5 à $0,75 \mathrm{mg} / \mathrm{kg}$ pour les quatrième à sixième infusions [20].

Cinq de ces études [17-21] ont utilisé un schéma en ouvert. Elles ont toutes démontré une efficacité de la kétamine avec des taux de réponse variant entre 29 et $91 \%$ à la fin des 2 à 3 semaines d'infusions répétées. Le taux de réponse après la première infusion était cependant très faible, variant entre 7 et $25 \%$, suggérant un effet antidépresseur cumulatif dû à la répétition des infusions. Ces résultats contrastant avec ceux obtenus dans les études unidoses montrant un taux de réponse élevé, pourraient s'expliquer par le fait que ces cinq études permettaient aux patients de poursuivre leurs psychotropes, ce qui n'était pas le cas dans les études unidoses [7,
8, 13] ou dans l'étude de maintien [16]. La durée de l'effet antidépresseur à la fin de la série d'infusions était d'environ deux semaines pour la plupart de ces études.

La dernière de ces six études [22] est, à notre connaissance, la seule étude clinique randomisée-contrôlée de traitement de maintien et la première étude négative portant sur les effets antidépresseurs de la kétamine. Vingt-six patients présentant une dépression résistante au traitement et des idées suicidaires chroniques ont été recrutés. Aucune différence statistique entre le traitement par la kétamine et la prise du placébo (solution saline) n'a été observée, avec, respectivement, $25 \%$ et $33 \%$ de réponse. Néanmoins, l'échec de cette étude pourrait s'expliquer par les différences de sévérité de la dépression, sa chronicité, la taille réduite de l'échantillon de patients, un taux élevé d'abandon ( $31 \%$ dans chacun des groupes), ainsi que l'utilisation concomitante d'autres psychotropes pouvant diminuer la réponse à la kétamine. 
Une étude en 3 phases de Phillips et al., publiée en 2019 [23], marque la transition entre les travaux examinant l'efficacité de la kétamine à court terme et ceux cherchant à établir des stratégies de maintien à long terme. La phase 1 (incluant 43 patients) a reproduit les effets antidépresseurs d'une dose unique de kétamine versus le midazolam (Figure $1 \mathrm{~A}$ ). Après la rechute dépressive, les patients procédaient à la phase 2, au cours de laquelle ils recevaient 6 infusions de kétamine $(0,5 \mathrm{mg} / \mathrm{kg})$ administrées 3 fois par semaine. À chaque infusion, une proportion grandissante de patients atteignait le seuil de réponse ( $59 \%$ ) et de rémission ( $23 \%$ ) à la fin de cette phase (Figure 1B). Les patients ayant obtenu une réponse à la fin de la phase 2 (23 patients) poursuivaient alors l'étude avec la phase 3 qui consistait en quatre infusions hebdomadaires de kétamine. Avec ce traitement, le niveau de symptômes dépressifs est demeuré stable chez ces patients pendant ces 4 semaines (Figure 1C). II s'agissait de la première étude, outre une publication d'une série de cas [1], démontrant véritablement un maintien de la réponse antidépressive au-delà de la première série de traitements aigus.

\section{L'eskétamine}

Certaines évidences suggèrent que l'énantiomère $S$ de la kétamine serait le plus actif, notamment de par sa plus forte affinité pour le récepteur NMDA [24]. Cela, ainsi que la recherche d'une stratégie d'administration plus aisée pour le patient, a conduit au développement de l'eskétamine, une formulation à administration intra-nasale (IN) de la S-kétamine. Bien que controversée dans certaines études [25, 26], l'eskétamine a récemment été approuvée par la FDA (Food and Drug Administration) aux États-Unis, l'Agence européenne des médicaments (AEM) en Europe et Santé Canada pour le traitement de la dépression résistante au traitement.

Quatre études de phases II et III, conduites chez une population de patients adultes, ont utilisé des doses variables d'eskétamine (entre 28 et $84 \mathrm{mg}$ ) administrées 2 fois par semaine durant 2 à 4 semaines [2730]. Pour trois d'entre elles [28-30], le schéma expérimental comparait l'association de l'eskétamine ou d'un placébo au standard de soins, consistant à débuter et optimiser un médicament oral à action antidépressive. Deux d'entre elles (une de phase II et une de phase III) ont démontré un effet statistiquement supérieur pour l'eskétamine selon le critère principal d'évaluation ${ }^{5}$. Toutefois, une méta-analyse réalisée par Zheng et al. [31] qui regroupe les données de ces 4 études, avec un total de 708 patients dont 419 ayant reçu l'eskétamine, montre que son effet est statistiquement supérieur à celui du placébo pour la réponse clinique mais aussi pour la rémission jusqu'à 28 jours.

Une étude de phase III s'est intéressée aux patients gériatriques [32]. Elle n'a pas démontré d'effet significatif pour le critère d'évaluation principal (amélioration clinique au jour 28). Néanmoins, des sousanalyses ont révélé un effet pour les patients de 74 ans et moins, mais pas pour les personnes âgées de 75 ans et plus. Ceci pourrait s'expli-

5 Selon les études, le critère principal était défini comme la réponse à $4 \mathrm{~h}$ post infusion, ou au jour 8 post infusion ou au jour 28 post infusion. quer par une physiopathologie de la dépression qui diffère dans ce groupe d'âge, avec un effet différentiel de l'eskétamine selon la physiopathologie à l'origine des symptômes dépressifs.

La combinaison d'eskétamine et d'un médicament oral à action antidépressive semble, dans une étude portant sur un groupe de 297 patients [33], supérieure à une combinaison avec un placébo pour prévenir la rechute dépressive pour une population ayant atteint une réponse, ou une rémission clinique stable, traitée par l'eskétamine associée à un médicament oral : une diminution du risque de rechute de $51 \%$ pour les patients en rémission, et $70 \%$ pour ceux ayant atteint une réponse a pu être observée (Figure 2).

\section{Les effets secondaires de la kétamine}

Les effets secondaires les plus fréquents de la kétamine sont les effets psychoactifs et cardiovasculaires. Plusieurs des études prospectives ont évalué les effets dissociatifs et psychotomimétiques à l'aide d'échelles standardisées. En raison d'une augmentation de noradrénaline circulante, causée par l'inhibition des pompes permettant l'entrée de la noradrénaline dans les cellules, une augmentation transitoire et bénigne de la tension artérielle et, parfois, de la fréquence cardiaque a pu être observée. Dans tous les cas, ces effets secondaires sont de courtes durées, généralement résolus en moins de deux heures, souvent en moins de 30 minutes. Bien avant la démonstration des effets antidépresseurs de la kétamine, des doutes étaient apparus en raison de son potentiel effet addictif [34]. Des toxicités vésicales et des déficits de mémoire sémantique et épisodique, chez les utilisateurs chroniques de kétamine, utilisée comme drogue en l'absence de prescription médicale ont également été rapportés [35]. Jusqu'à présent, ces effets secondaires n'ont cependant pas été actés dans la littérature.

\section{Potentiels mécanismes d'action antidépressive de la kétamine}

Le principal mécanisme d'action de la kétamine serait son rôle d'antagoniste non compétitif des récepteurs glutamatergiques de type NMDA. Ses propriétés anesthésiques, analgésiques et antidépressives impliqueraient principalement ce mécanisme [24]. Rappelons que le glutamate est le principal neurotransmetteur excitateur du système nerveux central. II est étroitement contrôlé, une surexcitation glutamatergique pouvant être à l'origine d'une neurotoxicité. Deux catégories de récepteurs glutamatergiques existent: 


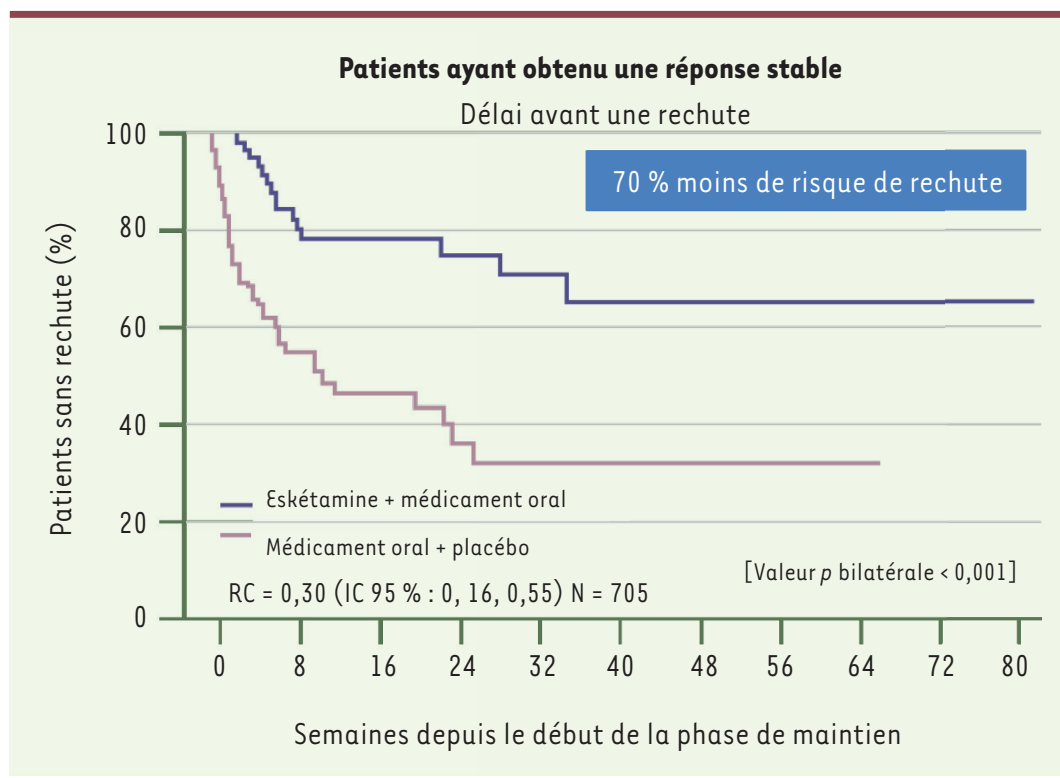

Figure 2. Les patients devaient avoir atteint et maintenu une réponse, soit une diminution de $50 \%$ ou plus sur l'échelle MADRS (Montgomery-Åsberg Depression Rating Scale) pour 2 visites consécutives à la fin d'une période de maintien de 16 semaines. Une rechute est définie : 1) par l'obtention d'un pointage total de 22 ou plus sur le MADRS pour 2 visites consécutives espacées de 5 à 15 jours, 2) une hospitalisation due à une détérioration, 3) une tentative de suicide, ou 4) tout évènement cliniquement significatif suggérant une rechute. Le temps médian pour que $50 \%$ des patients rechutent avec le retrait de l'eskétamine a été de 273 jours (non estimable avec la prolongation de l'eskétamine). Médicament oral: inhibiteur sélectif de recapture de sérotonine (ISRS) ou inhibiteur de recapture de noradrénaline et sérotonine (IRNS) à doses antidépressives; RC : risque cumulatif ; IC : intervalle de confiance $95 \% ; N$ : nombre total de patients randomisés avec une distribution 1:1 (adapté de [33]).

les récepteurs glutamatergiques ioniques, qui comprennent les récepteurs AMPA (acide $\alpha$-amino-3-hydroxy-5-méthyl-isoxazole4 -propionique), les récepteurs NMDA et kaïnate; et les récepteurs métabotropiques couplés aux protéines $G$. Les récepteurs AMPA sont perméables aux cations (sodium et potassium). Leur ouverture par le glutamate entraîne une dépolarisation membranaire qui permet de déloger le magnésium qui bloque, à l'état de repos, le canal du récepteur NMDA. Cette libération du canal permet au glutamate d'activer à son tour, avec le coactivateur glycine ou D-sérine, le récepteur NMDA, provoquant un influx de calcium responsable d'une série de cascades intracellulaires. L'action des récepteurs AMPA et NMDA est donc complémentaire. Ensemble, ils contribuent aux processus de plasticité synaptique [36]. La kétamine se loge, avec une haute affinité en présence du magnésium, dans le site PCP (phéncyclidine) du récepteur NMDA localisé à l'intérieur du canal ionique [24].

L'hypothèse que l'action antidépressive rapide de la kétamine reposait sur un effet activateur, préférentiel en sa présence, du glutamate sur les récepteurs AMPA versus les récepteurs NMDA (Figure 3) a été émise par Maeng et Zarate [36]. La kétamine, possiblement en bloquant les récepteurs NMDA des interneurones inhibiteurs GABA (gamma-aminobutyric acid) [37], provoquerait une augmentation de la libération de glutamate dans certains circuits neuronaux. Cette augmentation de glutamate dans la fente synaptique, les récepteurs NMDA étant bloqués, résulterait en une activation préférentielle des récepteurs AMPA [36-38]. II en découlerait une altération de diverses cascades intracellulaires impliquées dans la neuroplasticité, telles que les voies de signalisation de mTORCl (mechanistic target of rapamycin complex 1 ) et du BDNF (brain-derived neurotrophic factor), et une augmentation de la transcription des gènes codant les récepteurs AMPA [39], conduisant à un accroissement de la synaptogenèse.

En plus de son activité antagoniste des récepteurs NMDA, de nombreux travaux ont montré l'action de la kétamine, et/ou de ses métabolites, sur d'autres cibles moléculaires. Parmi celles-ci, notons les récepteurs $D_{2}$ (dopaminergiques de type 2), les récepteurs de la sérotonine $5-\mathrm{HT}_{2}$ (5-hydroxytryptamine de type 2) et $5-\mathrm{HT}_{18}$, des opioïdes, sigma, cholinergiques muscariniques (M1, M2, M3) et nicotiniques, GABA, de même que certains canaux sodiques et calciques (une revue exhaustive traitant de ce sujet pourra être consultée dans la revue de Zanos et al. [24]). L'étude des effets pharmacodynamiques de la kétamine est rendue difficile car des effets différentiels entre la kétamine racémique, la S-kétamine, la R-kétamine, et plusieurs de ses métabolites ont été rapportés et cela vis-à-vis des différentes cibles moléculaires. II est en effet possible que certaines propriétés de la kétamine qui sont rapportées découlent en partie de ses composés racémiques et/ou de ses métabolites actifs. Les constantes de dissociation de la kétamine et de ses métabolites pour les différentes cibles moléculaires étudiées sont généralement élevées. Leurs significations physiologiques aux doses subanesthésiques utilisées pour traiter la dépression restent donc incertaines dans la plupart des cas. Il est, par ailleurs, souvent difficile de déterminer si l'effet observé sur les différentes cibles moléculaires est un effet direct de la kétamine (ou de ses métabolites), ou indirect, découlant des effets antagonistes exercés sur les récepteurs NMDA. À noter que la kétamine possède également des propriétés anti-inflammatoires [24]. Mais les relations entre anti-inflammation et propriétés antidépressives de la kétamine restent à être clarifiées. 


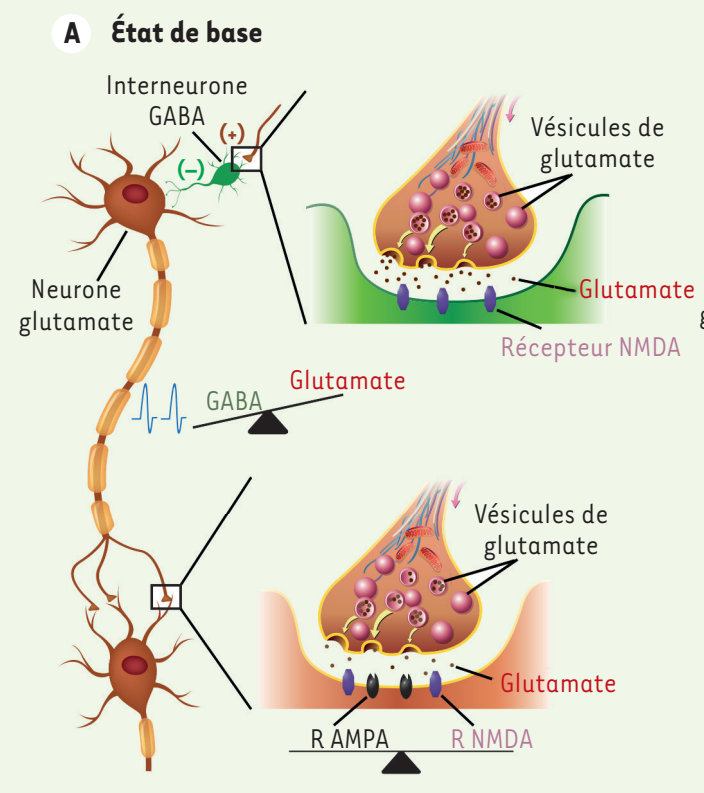

B Kétamine

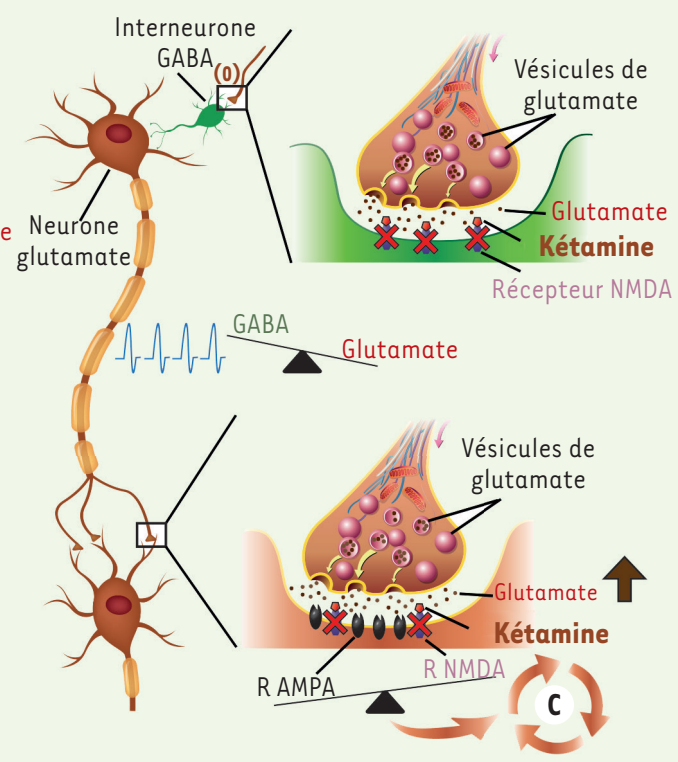

Figure 3. Représentation schématique de l'hypothèse de Maeng et Zarate. A. À l'état de base, le glutamate active des récepteurs NMDA (N-méthylD-aspartate) sur des interneurones GABA (gamma-aminobutyric acid), inhibant l'activité des neurones cibles, diminuant ainsi la libération de glutamate par ces derniers. $B$. En présence de la kétamine, l'inhibition des interneurones est levée, augmentant la libération de glutamate. Puisque les récepteurs NMDA sont bloqués par la kétamine, l'activité différentielle des récepteurs AMPA ( $\alpha$-amino-3-hydroxy-5-methylisoxazole-4-propionic acid) se trouve augmentée. $C$. Une série de cascades intracellulaires se trouvent altérées. $R$ : récepteur ; (+) : activation ; $(-)$ : inhibition ; $(0)$ : le signal est bloqué. L'intensité de l'activité électrophysiologique du neurone induite par le glutamate est indiquée par le nombre de potentiels d'action en bleu.

\section{La kétamine et l'idéation suicidaire}

Plusieurs études ont rapporté une diminution des idées suicidaires après un traitement par la kétamine. Une méta-analyse publiée en 2020 [40], a compilé les données de 25 publications issues de 15 études indépendantes incluant, au total, 572 participants. De cette étude, une réduction significative des idées suicidaires a pu être notée jusqu'à 72 heures après une injection unique de kétamine. Les résultats de ces différentes études restaient cependant très hétérogènes. Récemment, Phillips et al. [41] ont cependant rapporté une diminution prolongée des idées suicidaires après l'administration répétée de kétamine. II s'avère donc que de nouvelles recherches sont nécessaires afin de mieux caractériser les propriétés anti-suicidaires de la kétamine et son utilité clinique.

\section{Le maintien de la réponse antidépressive... à long terme}

Une question importante qui demeure est celle du traitement nécessaire pour que soit maintenue la réponse à long terme. Jusqu'à présent, la meilleure stratégie semble être l'administration répétée d'infusions de kétamine $[1,23,33]$. Seules trois études, qui ont échoué, ont exploré une stratégie de prévention de la rechute avec l'administration quotidienne d'une médication orale (2 études avec le riluzole, un agent modulateur du glutamate, et une étude avec le lithium). II est donc nécessaire de poursuivre la recherche afin de comprendre quelles associations de psychotropes pourraient augmenter ou diminuer [42] la réponse clinique à la kétamine.

L'étude CANBIND-13 [43] est actuellement en cours. II s'agit d'une large étude canadienne multicentrique, qui devrait apporter des données importantes quant à l'administration à long terme de la kétamine. Le principal objectif de cette étude est de comparer l'efficacité d'un traitement aigu de kétamine à l'électroconvulsivothérapie. Un objectif secondaire est de déterminer le temps de rechute lors d'un espacement graduel des infusions d'entretien de kétamine pendant 6 mois. Une étude similaire, l'étude ELEKT-D [44] également en cours, compare, en une seule phase, un traitement aigu de kétamine à un traitement aigu d'électroconvulsivothérapie, sans phase de traitement d'entretien par la kétamine.

Une étude multicentrique portant sur le traitement à long terme avec l'eskétamine (clinicaltrials.gov NCT02782104) est également en cours. Elle devrait fournir des indications quant à la fréquence idéale des traitements d'entretien. 


\section{Quelles directions futures?}

Depuis la publication initiale du groupe de l'université de Yale en 2000, les connaissances portant sur l'effet antidépresseur de la kétamine ont grandement avancé. Pourtant, de nombreuses inconnues demeurent et la recherche se doit de poursuivre les efforts afin d'élucider, entre autres, les stratégies optimales de maintien de la réponse, l'innocuité à long terme, ainsi que les mécanismes d'action de la kétamine. Alors que déjà les traitements hors monographie (en cliniques privées) par la kétamine se multiplient aux États-Unis et au Canada, les réponses à ces questions seront importantes pour assurer la qualité des soins offerts aux patients. Aucune étude de comparaison entre kétamine et eskétamine n'a été réalisée à ce jour. Pourtant, la question du «meilleur agent » ou du meilleur ratio «coût-bénéfice » sera sans aucun doute soulevée pour la planification des soins. Et finalement, notons que la découverte de l'action antidépressive de la kétamine a permis d'ouvrir la porte à la recherche d'autres molécules ciblant le système glutamatergique, qui possiblement apportera une réponse encore plus importante, plus durable, et avec moins d'effets secondaires que celle que donne la kétamine. $\diamond$

\section{SUMMARY}

\section{A review of the antidepressant properties of ketamine}

Major depression is a frequent and disabling disorder. Despite great developments in the field of psychopharmacology since the 1950's, delayed onset of action and treatment resistance to current pharmacological options, such as serotonin reuptake inhibitors, remain a therapeutic challenge. The recent discovery of the rapid antidepressant action of ketamine, an NMDA ( $\mathrm{N}$-methyl-D-aspartate) receptor antagonist, has brought a revolution to this field. This paper presents a comprehensive review of the clinical research on the antidepressant properties of ketamine as well as its presumed mechanisms of action. $\diamond$

\section{LIENS D'INTÉRÊT}

Pierre Blier a reçu des honoraires pour des présentations et/ou pour sa participation à des conseils consultatifs et/ou pour témoignage d'expert pour Allergan, Bristol Myers Squibb, Janssen, Lundbeck/Otsuka, et Pfizer. II a reçu des fonds de recherche de l'Institut de recherche en santé du Canada, Canadian Biomarkers for Depression, et Ontario Brain Institute et a participé à deux essais cliniques sur la eskétamine. Charles Desfossés a reçu une subvention pour études postdoctorales (fellowship) de Janssen.

\section{RÉFÉRENCES}

1. Archer S, Chrenek C, Swainson J. Maintenance ketamine therapy for treatment-resistant depression. J Clin Psychopharmacol 2018 ; 38 : 380-4.

2. World health organization. The global burden of disease: a 2004 update. Geneva : WHO Press, 2008.

3. Pereira VS, Hiroaki-Sato VA. A brief history of antidepressant drug development: from tricyclics to beyond ketamine. Acta Neuropsychiatr 2018 ; 30 : 307-22.

4. Blier P. Rational site-directed pharmacotherapy for major depressive disorder. Int J Neuropsychopharmacol $2014 ; 17: 997-1008$.

5. Rush AJ, Trivedi MH, Wisniewski SR, et al. Acute and longer-term outcomes in depressed outpatients requiring one or several treatment steps: a Star*D report. Am J Psychiatry 2006; 163 : 1905-17.

6. Skolnick P, Popik P, Trullas R. Glutamate-based antidepressants: 20 years on. Trends Pharmacol Sci $2009 ; 30: 563-9$.

7. Berman RM, Cappiello A, Anand A, et al. Antidepressant effects of ketamine in depressed patients. Biological Psychiatry $2000 ; 47: 351-4$.
8. Zarate CA, Jr., Singh JB, Carlson PJ, et al. A randomized trial of an N-methyl$D$-aspartate antagonist in treatment-resistant major depression. Arch Gen Psychiatry $2006 ; 63: 856-64$.

9. Diazgranados N, Ibrahim L, Brutsche NE, et al. A randomized add-on trial of an $\mathrm{N}$-methyl-D-aspartate antagonist in treatment-resistant bipolar depression. Arch Gen Psychiatry $2010 ; 67: 793-802$.

10. Zarate CA, Jr., Brutsche NE, Ibrahim L, et al. Replication of ketamine's antidepressant efficacy in bipolar depression: a randomized controlled addon trial. Biol Psychiatry $2012 ; 71$ : 939-46.

11. Ibrahim L, Diazgranados N, Luckenbaugh DA, et al. Rapid decrease in depressive symptoms with an $\mathrm{N}$-methyl-d-aspartate antagonist in $\varepsilon C T$ resistant major depression. Prog Neuropsychopharmacol Biol Psychiatry $2011 ; 35: 1155-9$.

12. Dale RM, Bryant KA, Thompson NR. Metabolic syndrome rather than body mass index is associated with treatment response to ketamine infusions.) Clin Psychopharmacol $2020 ; 40: 75-9$.

13. Murrough JW, losifescu DV, Chang LC, et al. Antidepressant efficacy of ketamine in treatment-resistant major depression: a two-site randomized controlled trial. Am J Psychiatry 2013 ; 170 : 1134-42.

14. Newport DJ, Carpenter LL, McDonald WM, et al. Ketamine and Other NMDA antagonists: early clinical trials and possible mechanisms in depression. Am J Psychiatry $2015 ; 172:$ 950-66.

15. Aan Het Rot M, Collins KA, Murrough JW, et al. Safety and efficacy of repeated-dose intravenous ketamine for treatment-resistant depression. Biol Psychiatry $2010 ; 67$ : 139-45.

16. Murrough JW, Perez AM, Pillemer S, et al. Rapid and longer-term antidepressant effects of repeated ketamine infusions in treatmentresistant major depression. Biol Psychiatry $2013 ; 74: 250-6$.

17. Rasmussen KG, Lineberry TW, Galardy CW, et al. Serial infusions of low-dose ketamine for major depression. J Psychopharmacol $2013 ; 27: 444-50$.

18. Diamond PR, Farmery AD, Atkinson $S$, et al. Ketamine infusions for treatment resistant depression: a series of 28 patients treated weekly or twice weekly in an ECT clinic. J Psychopharmacol $2014 ; 28$ : 536-44.

19. Shiroma PR, Johns B, Kuskowski M, et al. Augmentation of response and remission to serial intravenous subanesthetic ketamine in treatment resistant depression. J Affect Disord 2014 ; 155 : 123-9.

20. Cusin C, lonescu DF, Pavone KJ, et al. Ketamine augmentation for outpatients with treatment-resistant depression: Preliminary evidence for two-step intravenous dose escalation. Aust NZ J Psychiatry 2017 ; $51: 55-64$

21. Zheng W, Zhou YL, Liu WJ, et al. Rapid and longer-term antidepressant effects of repeated-dose intravenous ketamine for patients with unipolar and bipolar depression. J Psychiatr Res 2018 ; $106: 61-8$.

22. Ionescu DF, Bentley KH, Eikermann M, et al. Repeat-dose ketamine augmentation for treatment-resistant depression with chronic suicida ideation: a randomized, double blind, placebo controlled trial. J Affect Disord 2019 ; 243 : 516-24.

23. Phillips JL, Norris S, Talbot J, et al. Single, Repeated, and maintenance ketamine infusions for treatment-resistant depression: a randomized controlled trial. Am J Psychiatry 2019; 176: 401-9.

24. Zanos P, Moaddel R, Morris PJ, et al. Ketamine and ketamine metabolite pharmacology: insights into therapeutic mechanisms. Pharmacol Rev 2018 ; $70: 621-60$.

25. Cristea IA, Naudet F. US Food and Drug Administration approval of esketamine and brexanolone. Lancet Psychiatry $2019 ; 6: 975-7$.

26. Singh JB, Daly $\varepsilon$ J, Mathews $M$, et al. Approval of esketamine for treatmentresistant depression. Lancet Psychiatry $2020 ; 7: 232-5$.

27. Daly $\varepsilon$ J, Singh JB, Fedgchin M, et al. Efficacy and safety of intranasal esketamine adjunctive to oral antidepressant therapy in treatmentresistant depression. JAMA Psychiatry $2018 ; 75$.

28. Canuso CM, Singh JB, Fedgchin M, et al. efficacy and safety of intranasal esketamine for the rapid reduction of symptoms of depression and suicidality in patients at imminent risk for suicide: results of a double-blind, randomized, placebo-controlled study. Am J Psychiatry $2018 ; 175: 620-30$.

29. Fedgchin M, Trivedi M, Daly $\varepsilon$ J, et al. efficacy and safety of fixed-dose esketamine nasal spray combined with a new oral antidepressant in treatment-resistant depression: results of a randomized, double-blind, active-controlled study (Transform-1). Int J Neuropsychopharmacol 2019; $22: 616-30$.

30. Popova V, Daly $\varepsilon$ J, Trivedi M, et al. Efficacy and safety of flexibly dosed esketamine nasal spray combined with a newly initiated oral antidepressant in treatment-resistant depression: a randomized double-blind activecontrolled Study. Am J Psychiatry $2019 ; 176: 428-38$. 


\section{RÉFÉRENCES}

31. Zheng W, Cai DB, Xiang YQ, et al. Adjunctive intranasal esketamine for major depressive disorder: A systematic review of randomized double-blind controlled-placebo studies. J Affect Disord $2020 ; 265: 63-70$.

32. Ochs-Ross R, Daly $\varepsilon$ J, Zhang $Y$, et al. Efficacy and safety of esketamine nasal spray plus an oral antidepressant in elderly patients with treatment-resistant depression-Transform-3. Am J Geriatr Psychiatry $2020 ; 28: 121-41$.

33. Daly $\varepsilon$ J, Trivedi MH, Janik A, et al. Efficacy of esketamine nasal spray plus oral antidepressant treatment for relapse prevention in patients with treatment-resistant depression: a randomized clinical trial. JAMA Psychiatry $2019 ; 76: 893-903$.

34. Domino EF. Taming the ketamine tiger. 1965. Anesthesiology $2010 ; 113: 678-84$.

35. Short B, Fong J, Galvez V, et al. Side-effects associated with ketamine use in depression: a systematic review. Lancet Psychiatry $2018 ; 5: 65-78$.

36. Maeng $\mathrm{S}$, Zarate CA, Jr. The role of glutamate in mood disorders: results from the ketamine in major depression study and the presumed cellular mechanism underlying its antidepressant effects. Curr Psychiatry Rep 2007 ; 9 : 467-74.

37. Moghaddam B, Adams B, Verma A, Daly D. Activation of glutamatergic neurotransmission by ketamine: a novel step in the pathway from NMDA receptor blockade to dopaminergic and cognitive disruptions associated with the prefrontal cortex. J Neurosci 1997 ; 17 : 2921-7.

38. Maeng S, Zarate CA, Jr., Du J, et al. Cellular mechanisms underlying the antidepressant effects of ketamine: role of alpha-amino-3-hydroxy-5-methylisoxazole-4-propionic acid receptors. Biol Psychiatry $2008 ; 63: 349-52$.
39. Abdallah CG, Adams TG, Kelmendi B, et al. Ketamine's mechanism of action: a path to rapid-acting antidepressants. Depress Anxiety $2016 ; 33: 689-97$

40. Witt K, Potts J, Hubers A, et al. Ketamine for suicidal ideation in adults with psychiatric disorders: A systematic review and meta-analysis of treatment trials. Aust NZ J Psychiatry $2020 ; 54: 29-45$

41. Phillips JL, Norris S, Talbot J, et al. Single and repeated ketamine infusions for reduction of suicidal ideation in treatment-resistant depression. Neuropsychopharmacology $2020 ; 45: 606-12$.

42. Frye MA, Blier P, Tye SJ. Concomitant benzodiazepine use attenuates ketamine response: implications for large scale study design and clinica development. J Clin Psychopharmacol 2015 ; 35 : 334-6.

43. Phillips JL, Jaworska N, Kamler $\varepsilon$, et al. A randomized, crossover comparison of ketamine and electroconvulsive therapy for treatment of major depressive episodes: a Canadian biomarker integration network in depression (CAN-BIND) study protocol. BMC Psychiatry $2020 ; 20: 268$.

44. Mathew SJ, Wilkinson ST, Altinay M, et al. Electroconvulsive therapy (ect) vs. ketamine in patients with treatment-resistant depression: the Elekt-D study protocol. Contemp Clin Trials $2019 ; 77$ : 19-26.

\section{TIRÉS À PART}

C.Y. Desfossés

D ossédées du Malin au Moyen-Âge, les sorcières hystériques sont vouées au bûcher. Enfermées au XvII ${ }^{e}$ siècle, maltraitées, elles rejoignent la Cour des Miracles de l'Hospice de la Vieillesse-Femmes à la Salpêtrière... Jusqu'à ce que le Dr JeanMartin Charcot (1825-1893) mène le combat qui transforme l'ancien hospice en hôpital : l'École de la Salpêtrière de

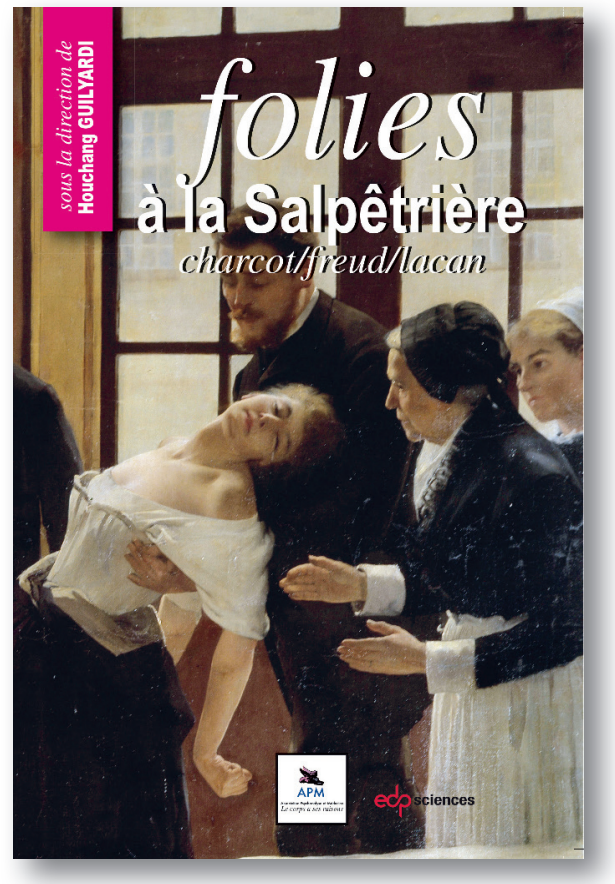

ISBN : 978-2-7598-1268-4
240 pages Paris est née, qui devient lieu de recherche, d'enseignement et de soins, de renommée internationale. Jean Martin Charcot n'a pas bonne presse, et pourtant... Hystérie et folie traversent les siècles, prenant les formes de «l'air du temps ».

De l'utérus migrateur d'Hippocrate aux recherches neurologiques de Charcot. Du désir inconscient avec Freud à la jouissance du parlêtre chez Lacan... C'est à cette traversée historique et conceptuelle que nous convie cet ouvrage.

Retrouvez toutes les Actualités de la Myologie sur les sites de :

la Société Française de Myologie www.sfmyologie.org la filière de santé neuromusculaire FILNEMUS www.filnemus.fr 